\section{ASTRONOMY}

\section{A galaxy far, far away}

Using the Hubble Space Telescope, astronomers have detected light from the most distant object yet found - a fledgling galaxy that existed when the Universe was just over 420 million years old. The discovery shows that stars were organizing into galaxies when the Universe was just 3\% of its current age of 13.7 billion years. Dan Coe of the Space Telescope Science Institute in Baltimore, Maryland, and his colleagues spotted light from the galaxy by taking advantage of an intervening lens of dark matter that magnified the object. Coe says that this and other discoveries suggest that light from galaxies might be responsible for ionizing the early Universe.

Astrophys J. 762, 32 (2013)

\section{Fine mind control of machine}

A woman paralysed in all four limbs has shown that she can move a prosthetic arm with unprecedented precision using only her brain to control the arm.

Andrew Schwartz at the University of Pittsburgh in Pennsylvania and his colleagues implanted two microelectrodes into the woman's motor cortex, the part of the brain responsible for initiating movement. The devices recorded cortical activity associated with her

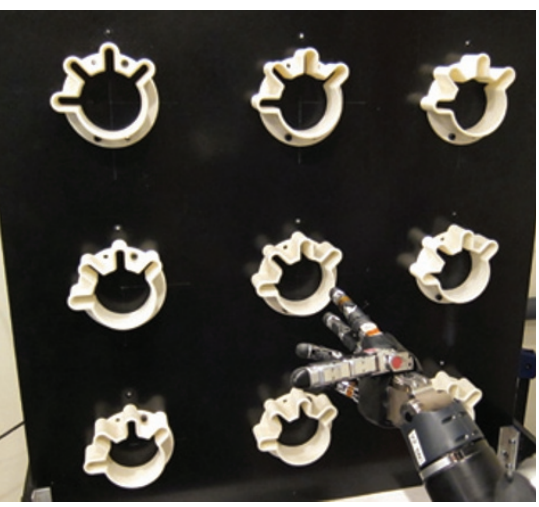

desire to move the robotic arm. An external decoder connected to the electrodes and the arm converted these signals into electronic instructions for the limb.

The woman began training to use the arm (pictured) - which has wrist-joint movement comparable to that of a human hand - less than two weeks after implantation. Within two days she could make simple movements, and within 13 weeks she could grasp and move objects almost as fast, smoothly and accurately as an able-bodied person.

Lancet http://dx.doi. org/10.1016/S01406736(12)61816-9 (2012)

\section{GENETIC ENGINEERING}

\section{Precise genomic editing}

A set of genetic tools based on components of a bacterial immune system can make the process of adding, deleting or replacing specific genes in model organisms and human cells easier and more precise.

Current gene-editing enzymes are expensive and difficult to use. Feng Zhang at the Broad Institute of MIT and Harvard in Cambridge, Massachusetts, and his colleagues developed an alternative method focusing on an enzyme, called Cas9, and two RNA molecules from a bacterial immune system known as CRISPR. These molecules work together to recognize and cut viral DNA. The team modified these molecules so that they could be used to edit human and mouse genomes at precise and even multiple locations.

George Church at Harvard Medical School in Boston,

Massachusetts, and his colleagues showed that their CRISPR-based geneediting system could be used in human cells, including reprogrammed stem cells. Science http://dx.doi. org/10.1126/science.1231143; http://dx.doi.org/10.1126/ science.1232033 (2013)

COMMUNITY CHOICE

The most viewed papers in science

\title{
Direct images of DNA
}

\section{Q HIGHLY READ \\ on pubs.acs.org in December}

Nearly 60 years after the structure of DNA was first discovered, scientists using transmission electron microscopy have captured direct images of it.

Enzo di Fabrizio at the Italian Institute of Technology in Genoa and his colleagues spread droplets of fluid containing DNA from a bacterium-infecting virus onto silicon wafers that were etched with micrometre-scale pillars and holes. As the droplets dried overnight, the DNA stretched taut across the tops of the pillars, passing over some of the holes. The researchers then imaged the DNA (pictured; scale bar, 20 nanometres) by beaming electrons through the holes.

The team observed a structure with helices that repeated roughly every 2.7 nanometres (inset, red arrows), consistent with A-DNA - a non-physiological DNA conformation known to occur in dehydrated samples.

Nano Lett. 12, 6453-6458 (2012)

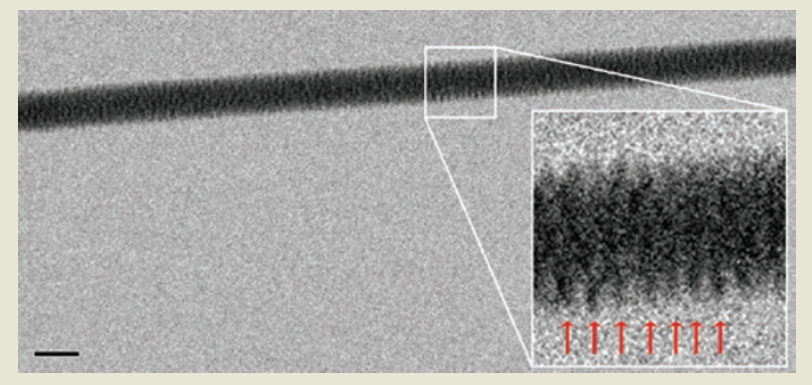

\section{Immune cells rejuvenated}

Two groups in Japan have reprogrammed cells that fight HIV and cancer, boosting the number and lifespan of the cells.

The body's immune system makes $T$ cells that target viruses and cancer, but the pool of these cells quickly becomes exhausted. Shin Kaneko and Hiromitsu Nakauchi at the University of Tokyo and their colleagues took a group of HIV-specific $T$ cells from a person with HIV. They reprogrammed the cells into stem cells, and then added key growth factors so that the stem cells developed into the original type of $\mathrm{T}$ cell. The rejuvenated cells retained their ability to target HIV, had an enhanced capacity for proliferation and had longer telomeres, or caps on the end of their chromosomes, suggesting a longer lifespan.

Hiroshi Kawamoto at the RIKEN Research Center for Allergy and Immunology in Yokohama and his colleagues used a similar approach to reprogram T cells specific to melanoma. The reprogrammed $\mathrm{T}$ cells could still recognize a protein commonly found on the surface of melanoma cells, and were present in greater numbers than they had been in the original population. Cell Stem Cell 12, 114-126; 31-36 (2013)

\section{$\rightarrow$ NATURE.COM}

For the latest research published by Naturevisit:

www.nature,com/latestresearch 